\title{
Is meridional circulation important in modelling irregularities of the solar cycle?
}

\author{
Bidya Binay Karak and Arnab Rai Choudhuri \\ Department of Physics, Indian Institute of Science, Bangalore 560012, India \\ email: bidya_karak@physics.iisc.ernet.in
}

\begin{abstract}
We explore the importance of meridional circulation variations in modelling the irregularities of the solar cycle by using the flux transport dynamo model. We show that a fluctuating meridional circulation can reproduce some features of the solar cycle like the Waldmeier effect and the grand minimum. However, we get all these results only if the value of the turbulent diffusivity in the convection zone is reasonably high.
\end{abstract}

Keywords. Sun: dynamo — Sun: activity — sunspots

\section{Introduction}

The solar cycle is not regular. There were several grand minima like the Maunder minimum during which the activity level was extremely low. Although the solar activity has varied approximately cyclically since the Maunder minimum, the amplitudes and the periods of individual cycles varied in an irregular manner. Another important feature of solar cycles is the Waldmeier effect, which is basically the anti-correlation between the rise time and the peak sunspot number. However, we define two different aspects of it, which we call WE1 and WE2. By WE1 we refer to the anti-correlation between the rise time and the peak sunspot number, whereas by WE2 we refer to the positive correlation between the rise rate and the peak sunspot number.

Our motivation is to model the irregularities of the solar cycle, including features like the Waldmeier effect, by using the flux transport dynamo model (see Choudhuri 2011 and references therein). In this model, the toroidal field is generated near the base of the convection zone by differential rotation and the poloidal field is generated near the solar surface by the decay of tilted bipolar sunspots. These two source regions are connected to each other by several transport agents. One important flux transport agent in this model is the turbulent magnetic diffusivity $\left(\eta_{t}\right)$. However, its value in the whole convection zone is not properly constrained. This has led to two different classes of models, in which the diffusivity has been taken to be high or low (see Jiang, Chatterjee \& Choudhuri 2007; Choudhuri 2011). The values of $\eta_{t}$ in these two classes of models are taken in the ranges $\sim 10^{12}-10^{13} \mathrm{~cm}^{2} \mathrm{~s}^{-1}$ and $\sim 10^{10}-10^{11} \mathrm{~cm}^{2} \mathrm{~s}^{-1}$ respectively. Another important flux transport agent in this model is the meridional circulation. The present understanding of its origin - and, more importantly, its fluctuations - is very primitive. It is believed that the the meridional circulation arises from a slight imbalance between two large terms (the centrifugal force due to the variation of angular velocity with distance from the equatorial plane and the thermal wind due to a temperature variation with latitude). Therefore, we expect that there may be random variations in the meridional circulation due to stochastic fluctuations in any one of these driving forces. Only since 1990s we have some observational data of meridional circulation near the surface. Therefore we do not know whether it had large variations in the past. However, we can get some idea of the variations of the meridional circulation from the observed periods of the past solar cycles. 


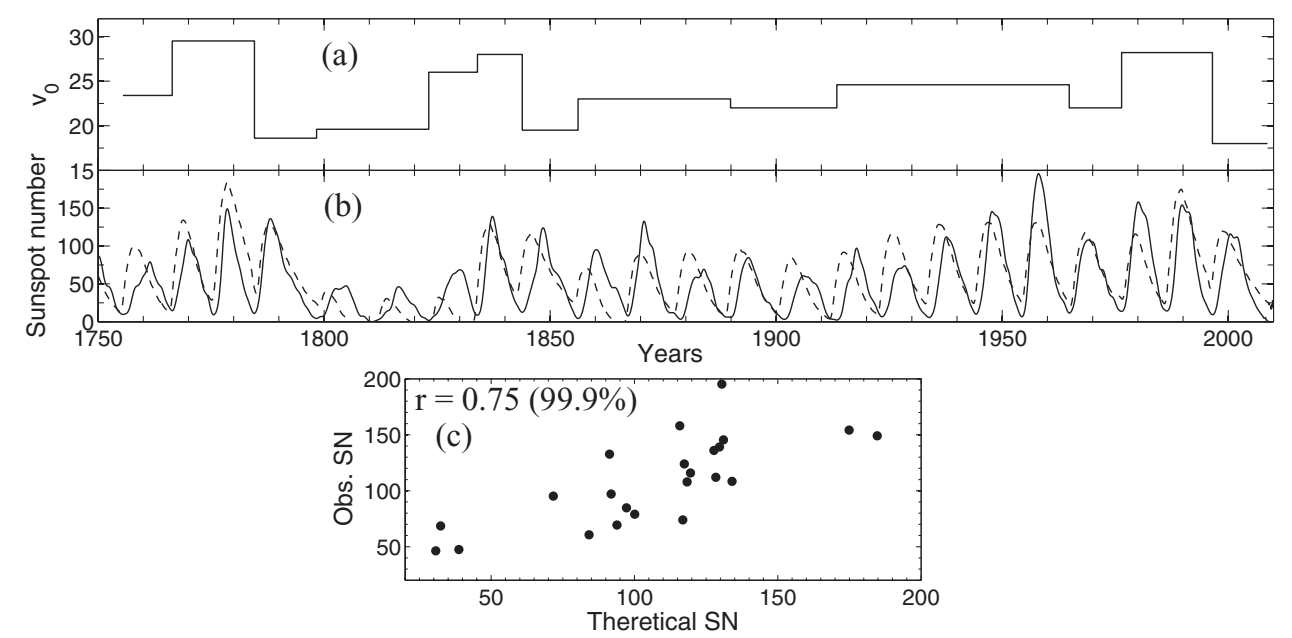

Figure 1. (a) Variation of amplitudes of meridional circulation $v_{0}$ (in $\mathrm{m} \mathrm{s}^{-1}$ ) with time (in yr). The solid line is the variation of $v_{0}$ used to match the theoretical periods with the observed periods. (b) Variation of theoretical sunspot number (dashed line) and observed sunspot number (solid line) with time. (c) Scatter diagram showing peak theoretical sunspot number and peak observed sunspot number. From Karak (2010).

In the next section, we discuss how this is done by using the flux transport dynamo model and then we model solar cycles using the variable meridional circulation.

\section{Methodology and results}

We know that the period of the cycle in the flux transport dynamo model is primarily determined by the strength of the meridional circulation (Dikpati \& Charbonneau 1999). A stronger meridional circulation makes the dynamo period shorter. Therefore it should be possible to match the observed periods of the last 23 solar cycles by using a variable meridional circulation. Karak (2010) performed this experiment using a high diffusivity model based on the model of Chatterjee, Nandy \& Choudhuri (2004). Fig. 1(a) shows the variation of the amplitude of meridional circulation required to match the periods of last 23 solar cycles. From this figure, we see that the meridional circulation varied significantly with time. Therefore, if the flux transport dynamo model is the correct model for the solar cycle, then we have to conclude that the meridional circulation had large variations in the past. Now let us look at the variation of theoretical sunspot number obtained by Karak (2010) shown by the dashed line in Fig. 1(b). For comparison, the observed sunspot number is shown by the solid line. Surprisingly, most of the theoretical sunspot cycle amplitudes are matched with the observed ones. We do get a good correlation between these two as shown in Fig. 1(c). This is a very important result of this analysis because our motivation was only to match the solar cycle periods and to get some idea of the variation of meridional circulation in the past. However, while doing this, we find that most of the solar cycle amplitudes are also matched to some extent. Therefore, this study suggests that a significant amount of fluctuations in the strengths of the cycles is arising from the variations in the meridional circulation, which seems important in modelling not only the solar cycle periods but also the cycle amplitudes. We point out that using a truncated dynamo model Passos (2012) (and references therein) have concluded that a variable meridional circulation is necessary in modeling irregular solar cycle. 

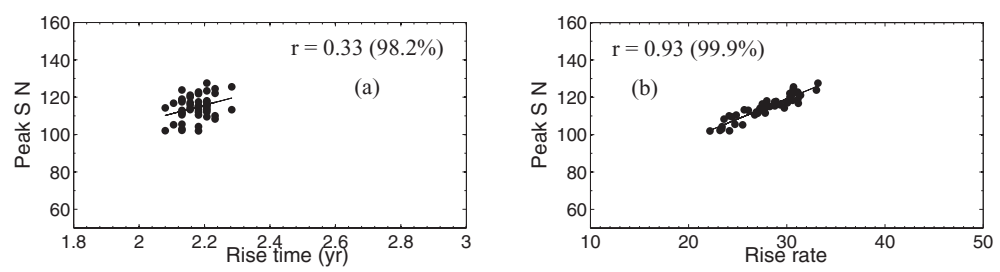

Figure 2. Results for WE1 (left panel) and WE2 (right panel) obtained by introducing fluctuations in the poloidal field at the minima. From Karak \& Choudhuri (2011).
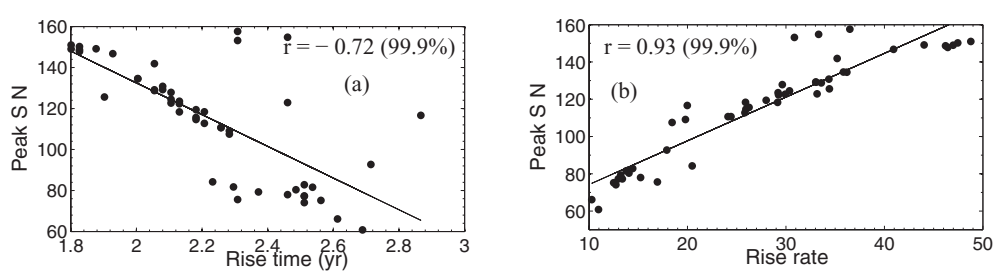

Figure 3. Same as earlier but obtained by introducing fluctuations in the meridional circulation. From Karak \& Choudhuri (2011).

Now let us explain the physics of this result based on the arguments given by Yeates, Nandy \& Mackay (2008). We know that in the flux transport dynamo, the production of the toroidal field is more if the poloidal field remains in the tachocline for longer time and vice versa. However, the poloidal field also diffuses during its transport through the convection zone. As a result, if the diffusivity is very high, then much of the poloidal field diffuses away and not much of it reaches the tachocline to induct the toroidal field. Therefore, when we decrease $v_{0}$ in high diffusivity model to match the period of a longer cycle, the poloidal field gets more time to diffuse during its transport through the convection zone. This ultimately leads to a lesser generation of toroidal field and hence the cycle becomes weaker. On the other hand, when we increase the value of $v_{0}$ to match the period of a shorter cycle, the poloidal field does not get much time to diffuse in the convection zone. Hence it produces stronger toroidal field and the cycle becomes stronger. Consequently, we get weaker amplitudes for longer periods and vice versa. However, this is not the case in low diffusivity model because in this model the diffusive decay of the fields is not very important. As a result, the slower $v_{0}$ means that the poloidal field remains in the tachocline for longer time and therefore it produces more toroidal field, giving rise to a strong cycle. Therefore, we do not get the correct correlation between the amplitudes of theoretical sunspot number and those of observed sunspot number when we repeat the same analysis in the low diffusivity model.

Next we present some results from Karak \& Choudhuri (2011), who studied the Waldmeier effect using the flux transport dynamo model. The stochastic fluctuations in the Babcock-Leighton process of generating poloidal field and the stochastic fluctuations in the meridional circulation are the two main sources of irregularities in this model. Therefore, to study the Waldmeier effect, we first introduce suitable stochastic fluctuations in the poloidal field source term. Fig. 2 shows the result. We see that this study cannot reproduce WE1 (Fig. 2(a)), although it reproduces WE2 (Fig. 2(b)). Next we introduce stochastic fluctuations in the meridional circulation. Fig. 3 shows this result. We see that both WE1 and WE2 are remarkably reproduced in this case. We may mention that very recently Pipin \& Kosovichev (2011) are also able to reproduce Waldmeier effect using their mean field dynamo model. 


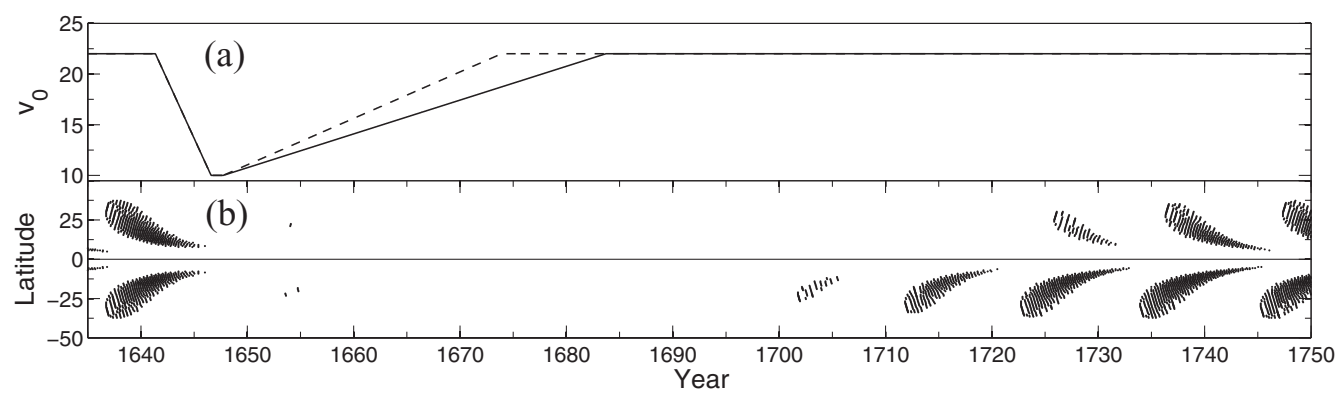

Figure 4. (a) The solid and dashed line show the variations of $v_{0}\left(\right.$ in $\mathrm{m} \mathrm{s}^{-1}$ ) in northern and southern hemispheres with time. (b) The butterfly diagram. From Karak (2010).

Karak (2010) found that a decrease of the meridional circulation to a very low value can reproduce a Maunder-like grand minimum. Fig. 4(a) shows the variation of $v_{0}$ required to model a Maunder-like grand minimum. Fig. 4(b) shows the butterfly diagram of sunspot numbers. This result reproduces most of the features of the Maunder minimum e.g., North-South asymmetry of sunspot (Sokoloff \& Nesme-Ribes 1994) and the sudden initiation of Maunder minimum (Usoskin, Mursula \& Kovaltsov 2000) remarkably well.

We have shown that the temporal variation of the meridional circulation is important in modelling irregularities of the solar cycle. Our studies also suggest that there have been large variations in the meridional circulation in the past. With suitable stochastic fluctuations in the meridional circulation, we are able to reproduce many important irregular features of the solar cycle including the Waldmeier effect and Maunder-like grand minima. However we fail to reproduce these results in a low diffusivity model. Therefore this study along with some other studies (Chatterjee, Nandy \& Choudhuri 2004; Chatterjee \& Choudhuri 2006; Jiang, Chatterjee \& Choudhuri 2007; Choudhuri \& Karak 2009; Goel \& Choudhuri 2009; Hotta \& Yokoyama 2010; Karak \& Choudhuri 2012) supports the high diffusivity model for the solar cycle.

\section{Acknowledgments}

We thank IAU funding agencies and DST, India, for financial support.

\section{References}

Chatterjee, P. \& Choudhuri, A. R. 2006, Solar Phys., 239, 29

Chatterjee, P., Nandy, D., \& Choudhuri, A. R. 2004, A\&A, 427, 1019

Choudhuri, A. R. 2011, Pramana, 77, 77

Choudhuri, A. R., Chatterjee, P., \& Jiang, J. 2007, Phys. Rev. Lett., 98, 1103

Choudhuri, A. R. \& Karak, B. B. 2009, RAA, 9, 953

Choudhuri, A. R., Schüssler, M., \& Dikpati, M. 1995, A\&A, 303, L29

Dikpati, M. \& Charbonneau, P. 1999, ApJ, 518, 508

Goel, A. \& Choudhuri, A. R. 2009, RAA, 9, 115

Hotta, H. \& Yokoyama, T. 2010, ApJ, 714, L308

Jiang, J., Chatterjee, P., \& Choudhuri, A. R. 2007, MNRAS, 381, 1527

Karak, B. B. 2010, ApJ, 724, 1021

Karak, B. B. \& Choudhuri, A. R. 2011, MNRAS, 410, 1503

Karak, B. B. \& Choudhuri, A. R. 2012, Solar Phys., 278, 137

Passos, D. 2012, ApJ, 744, 172

Pipin, V. V. \& Kosovichev, A. G. 2011, ApJ, 741, 1

Sokoloff, D. \& Nesme-Ribes, E. 1994, A\&A A, 288, 293 
Usoskin, I. G., Mursula, K., \& Kovaltsov, G. A. 2000, A\& A, 354, L33

Yeates, A. R., Nandy, D., \& Mackay, D. H. 2008, ApJ, 673, 544

\section{Discussion}

ANDREY TLATOV: Solar cycles overlap and changing meridional circulation may affect this overlap. Can you comment on this?

BIDYA KARAK: Yes, there is an overlap between two cycles. This overlap is affected by the meridional circulation speed. If the meridional circulation is weaker, then the overlap is less. Whereas, if the meridional circulation is stronger, then, the overlap is larger. This issue has been discussed by Nandi et al. (2011), Nature paper.

JANET LUHMANN: If meridional circulation changes are not stochastic but affected by active regions or sunspots, what are the consequences for the model?

BIDYA KARAK: There is an indication that the meridional circulation has a periodic variation with the solar cycle becoming stronger during solar minimum and weaker during solar maximum. It is not clear whether this variation is coming due to the active regions or due to the Lorentz force of the magnetic field acting on the velocity field (Karak \& Choudhuri 2012). As the meridional circulation is coming from a slight imbalance between two large terms, we expect the meridional circulation to vary cycle to cycle stochastically. Indeed from the variation of the observed periods of the solar cycle, we inferred this kind of stochastic variation. Our modeling is based on the stochastic variation of the meridional circulation. However, if the meridional circulation changes are only due to active regions, we cannot model the irregular solar cycle using this model. 